\title{
Demonstrating polymorphic miRNA-mediated gene regulation in vivo: Application to the $g+6223 G \rightarrow A$ mutation of Texel sheep
}

\author{
HARUKO TAKEDA, ${ }^{1}$ CAROLE CHARLIER, ${ }^{1}$ FRÉDÉRIC FARNIR, ${ }^{2}$ and MICHEL GEORGES ${ }^{1}$ \\ ${ }^{1}$ Unit of Animal Genomics, GIGA Research Center, Faculty of Veterinary Medicine, University of Liège, 4000-Liège, Belgium \\ ${ }^{2}$ Unit of Biostatistics, GIGA Research Center, Faculty of Veterinary Medicine, University of Liège, 4000-Liège, Belgium
}

\begin{abstract}
We herein describe the development of a biochemical method to evaluate the effect of single nucleotide polymorphisms (SNPs) in target genes on their regulation by microRNAs in vivo. The method is based on the detection of allelic imbalance in RNAs coimmunoprecipitated with AGO proteins from tissues of heterozygous individuals. We characterize the performances of our approach using a model system in a cell culture, and then apply it successfully to prove that the $3^{\prime}$ UTR $g+6223 G \rightarrow A$ mutation operates by promoting RISC-dependent down-regulation of myostatin (MSTN) in skeletal muscle of Texel sheep.
\end{abstract}

Keywords: AGO immunoprecipitation; allelic imbalance; microRNA target; polymorphic miRNA-mediated gene regulation

\section{INTRODUCTION}

The majority of mammalian genes are fine tuned by one or several of an arsenal of $\sim 600$ microRNAs (miRNAs) (e.g., Friedman et al. 2009). The sequence space devoted to miRNA-mediated gene regulation (i.e., miRNA precursors, miRNA target sites, silencing machinery) is bound to suffer its toll of DNA sequence variants, of which some will impact function and possibly phenotype. This assertion is supported by (1) strong genetic and functional evidence for the creation of an illegitimate miRNA target site in the 3'UTR of the MSTN gene by the Texel mutation associated with increased muscle mass in sheep (Clop et al. 2006); (2) genetic and functional evidence that naturally occurring (human) and ENU-induced (mouse) mutations in the seed sequence of miR-96 cause progressive hearing loss by affecting pre-miRNA processing and/or target recognition (Lewis et al. 2009; Mencía et al. 2009); (3) signatures of purifying selection on single nucleotide polymorphisms (SNPs) destroying conserved miRNA target sites (Chen and Rajewsky 2007); and (4) the observation of downward shifts in the relative transcript levels of the targeted versus

Reprint requests to: Michel Georges, Unit of Animal Genomics, GIGA Research Center, Faculty of Veterinary Medicine, University of Liège, B34, 1 Avenue de l'Hôpital, 4000-Liège, Belgium; e-mail: michel.georges@ ulg.ac.be; fax: 32-4-366-41-98.

Article published online ahead of print. Article and publication date are at http://www.rnajournal.org/cgi/doi/10.1261/rna.2131110. untargeted allele in tissues of mice heterozygous for SNPs that alter recognition sites for coexpressed miRNAs (Kim and Bartel 2009).

At least 10 associations have been reported between complex disease and $3^{\prime}$ UTR SNPs predicted to alter miRNA target sites (Abelson et al. 2005; Züchner et al. 2006; Adams et al. 2007; Mishra et al. 2007; Sethupathy et al. 2007; Tan et al 2007; Beetz et al. 2008; Brendle et al. 2008; Chin et al. 2008; Kapeller et al. 2008; Landi et al. 2008; Lv et al. 2008; Wang et al. 2008; Jensen et al. 2009). However, as pointed out by Sethupathy and Collins (2008), in most of these cases the evidence supporting the hypothesis was suggestive at best. Moreover, tens of thousands of common SNPs destroy or create putative miRNA target sites in the $3^{\prime}$ UTR of 12,300 human genes (e.g., Hiard et al. 2010). Yet, identifying the truly functional target sites, and thereby the relevant SNPs, remains a major challenge.

While it seems inescapable that polymorphic miRNAmediated gene regulation makes a significant contribution to phenotypic variation, there is a clear need for approaches that allow effective identification of the corresponding DNA sequence variants. We herein describe a method that achieves this goal for DNA sequence variants in $3^{\prime}$ UTRs. It is based on the detection of allelic imbalance in the product of RNA immunoprecipitation (RIP) from tissues of heterozygous individuals. We apply the method successfully to the MSTN 3'UTR mutation of Texel sheep, thereby formally proving its causality and modus operandi. 


\section{RESULTS}

\section{The model: The sheep Texel mutation}

Quantitative trait loci (QTL) analysis pinpointed a G-to-A transition in the $3^{\prime} \mathrm{UTR}$ of the MSTN gene associated with increased muscularity in sheep. The $g+6223 G-A$ mutation, originating from Texel sheep, was predicted to create an illegitimate 8-mer target site for coexpressed miR-1 and miR-206. Accordingly, mutant MSTN mRNA was found to be approximately one-third less abundant than wild-type MSTN mRNA in skeletal muscle of heterozygous animals (compatible with miRNA-dependent target degradation), while circulating levels of MSTN protein were found to be approximately two-thirds lower in homozygous mutants than in homozygous wild types (compatible with additional translational inhibition). When introduced in the $3^{\prime}$ UTR of the TK-driven luciferase gene, the $g+6223 G-A$ substitution caused an miR-1/miR-206-dependent reduction of luminescence by approximately two-thirds when present in four copies and by approximately one-third when present in one copy. These findings made a compelling case for enhanced muscularity caused by perturbed miRNA-mediated gene regulation of the MSTN gene. Yet, one could argue that the accumulated evidence did not formally prove our hypothesis; hence, having to qualify the target site as "potential" in the title (Clop et al. 2006).

To provide conclusive evidence in support of our hypothesis, we aimed at demonstrating that MSTN transcripts with the $g+6223 \mathrm{~A}$ mutation more tightly associate with the RNA-induced silencing complex (RISC) than with wildtype MSTN transcripts in vivo. To perform the comparison in optimally controlled conditions, we aimed at demonstrating differential RISC association by means of an allelic imbalance test in anti-AGO2 immunoprecipitate from skeletal muscle of animals heterozygous for the $g+6223 G$ A mutation (Fig. 1).

\section{Luciferase reporter transcripts with four tandem copies of the Texel mutation are preferentially coimmunoprecipitated by anti-AGO2 antibodies in a miR-1-dependent fashion}

To optimize conditions for RIP suiting our purpose we took advantage of the luciferase reporter assays previously developed to demonstrate the effect of the $g+6223 G-A$ mutation on miR-1-dependent gene regulation (Clop et al. 2006). The first assay relied on (1) the $p R L-T K-4 x A$ and $p R L-T K-4 x G$ vectors, characterized by four tandem copies of an 80-base-pair (bp) segment of the sheep MSTN 3'UTR encompassing the $g+6223 G-A$ site embedded in the $3^{\prime}$ UTR of the renilla luciferase reporter gene $(p R L-T K-4 x G$ contains the wild-type version of the $80-\mathrm{bp}$ repeat, while $p R L-T K-4 x A$ corresponds to the mutant one); and (2) the pcDNA3-miR-1 and pcDNA3-miR-377 vectors expressing ovine pri-miR-1 and $m i R-377$ (negative control), respectively

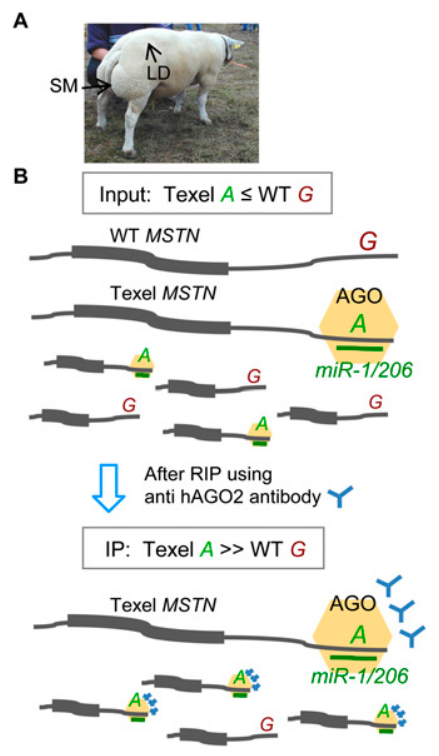

FIGURE 1. (A) Texel sheep with muscular hypertrophy resulting in part from the $g+6223 G-A$ mutation in the $3^{\prime}$ UTR of the MSTN gene. The approximate positions of the semimembranosus (SM) and longissimus dorsi (LD) muscle examined in this study are shown. (B) Schematic representation of the allelic imbalance test developed in this study to demonstrate the preferential association of the mutant MSTN $A$ allele over the wild-type MSTN $G$ with the RISC complex. Prior to immunoprecipitation, transcripts carrying the $G$ allele are predicted to be slightly more abundant than those carrying the $A$ allele in skeletal muscle of heterozygous $A / G$ animals as a result of the RISC-dependent degradation of the targeted $A$ transcripts. The coimmunoprecipitation of miRNA-regulated mRNA with antibodies directed against RISC components is predicted to cause a net enrichment in targeted $A$ transcripts.

(Fig. 2A). We previously demonstrated that cotransfection of $p c D N A 3-m i R-1$ with $p R L-T K-4 x A$, but not with $p R L-T K-$ $4 x G$, reduced luciferase activity approximately threefold when compared with cotransfection with pcDNA3-mir-377 or an empty pcDNA3 vector (Clop et al. 2006).

In addition to these available vectors, we derived $p c D N A 3-$ $m i R-1 M$ from $p c D N A 3-m i R-1$ by mutagenizing the $\mathrm{T}$ at position six of miR-1 into C. pcDNA3-miR-1M is predicted to drive expression of a miRNA ( $m i R-1 M)$ that targets the wild-type $(G)$ but not Texel (A) MSTN 3'UTR sequence, i.e., the opposite of miR-1 (Fig. 2B). Indeed, cotransfecting $p R L$ $T K-4 x G$ (wild-type allele) with $p c D N A 3-m i R-1 M$ caused an approximately fourfold reduction in luminescence when compared with cotransfection with pcDNA3-miR-1 (Fig. $2 C)$. As a matter of fact, the effect of $m i R-1 M$ on the wildtype $G$ allele (approximately fourfold reduction) was slightly stronger than that of $m i R-1$ on the Texel $A$ allele (approximately threefold reduction). This could be due to the substitution of an $\mathrm{A}-\mathrm{U}$ base pair with a more stable $\mathrm{G}-\mathrm{C}$ base pair in the miRNA target duplex (Fig. 2B,C).

To mimic heterozygosity for the $g+6223 G-A$ mutation, we cotransfected HeLa cells with (1) equimolar amounts of the $p R L-T K-4 x A$ and $p R L-T K-4 x G$ vectors; and (2) pcDNA3 vectors expressing either $m i R-1$ (targeting the $4 x A$ transcripts), 
$m i R-1 M$ (targeting the $4 x G$ transcripts), or $m i R-377$ (not targeting either transcript). As expected, luciferase activity was reduced approximately twofold when cotransfecting with either $p c D N A 3-m i R-1$ or $p c D N A 3-m i R-1 M$ when compared with $p c D N A 3-m i R-377$ (Fig. 2D). We then performed RIP on the corresponding cell extract using the 2A8 anti-human AGO2 monoclonal antibody (Nelson et al. 2007) (AGO-RIP), as well as with a control nonimmune mouse IgG (IgGRIP). Western blot analysis performed on input, supernatant, and immunoprecipitated material confirmed the immuno-enrichment of an $\sim 95-\mathrm{kDa}$ species, corresponding to human AGO proteins, when using the $2 \mathrm{~A} 8$, but not with the control antibody (Fig. 2E). Note that the 2A8 antibody was raised against human AGO2, yet recognizes the four human AGO proteins (AGO1-AGO4) in Western blot analysis (Nelson et al. 2007). We isolated RNA from input and immunoprecipitated material and performed RT-PCR amplification of vector-derived transcripts using primers flanking the $4 \times 80$-bp tandem repeat insertions. We consistently obtained slightly higher PCR yields from AGORIP of samples transfected with $m i R-1$ or $m i R-1 M$ when compared either with AGO-RIP of samples transfected with $m i R-377$ or with control IgG-RIP of samples transfected with either of the three miRNAs. This suggested that vector-derived transcripts were indeed enriched by the $2 \mathrm{~A} 8$ antibody in an $m i R-1 / m i R-1 M$-dependent manner. We directly sequenced the PCR products and estimated the allelic ratio from the peak heights corresponding to the $G$ and $A$ allele using Peakpicker (Ge et al. 2005). The quantitativeness of the method was demonstrated by generating a calibration curve using mixtures of the plasmid DNAs (Supplemental Fig. 1A). We reproducibly observed (1) close to equal $\mathrm{A} / \mathrm{G}$ ratios in all input samples, the AGO-RIP of miR-377 transfected samples, and all IgG-RIP samples; (2) an $\sim 2.6$-fold enrichment of $4 x A$ (Texel) product after cotransfection with $p c D N A$ miR-1 (overnight incubation with 2A8); and (3) an $\sim 4$.5-fold enrichment of $4 x G$ (wild-type) product after cotransfection with $p c D N A-m i R-1 M$ (Fig. 2F).
This was exactly as expected, assuming preferential $m i R$ 1 -mediated RISC targeting of the $4 x A$ transcripts, preferential $m i R-1 M$ mediated RISC targeting of the $4 x G$ transcripts, and no RISC targeting of either transcript by miR-377.

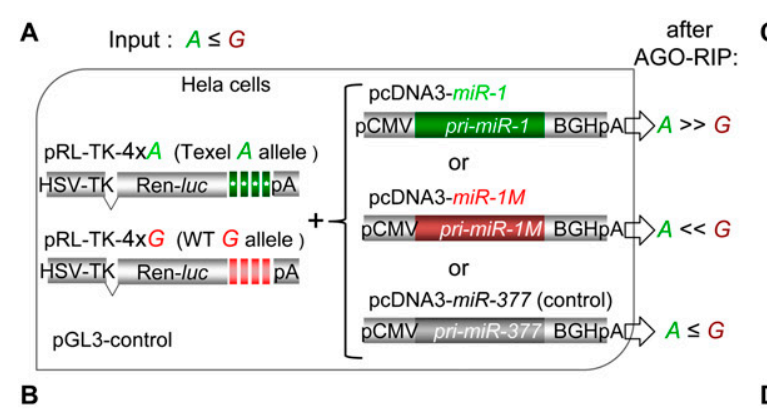

Texel MSTN 3' UTR : 5' UACUGUCAUUgUaUUCAAAUCUCAACAUUCCAUUAUUUUAAUA

$$
\begin{array}{cc}
\text { miR-1 : } & \text { II: I III IIIIIIII } \\
& \text { 3' AUGUAUGAAGAAUGUAGGU } \\
\text { miR-1M : } & \text { II: I III II IIIII } \\
& \text { 3' AUGUAUGAAGAAAUGCAAGGU }
\end{array}
$$

WT MSTN 3' UTR : 5' UACUGUCAUUGUAUUCAAAUCUCAACG̈UUCCAUUAUUUUAAUA $\begin{array}{ccc} & \text { II: I III II: IIIII } \\ \text { miR-1 : } & \text { 3' AUGUAUGAAGAAUGUAGGU }\end{array}$ $\begin{array}{ll}\text { miR-1M : } & \text { II: I III IIIIIIII } \\ \text { 3' AUGUAUGAAGAAAUGCAGGU }\end{array}$

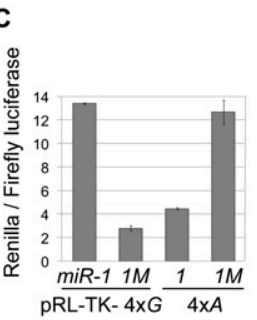

\section{D}

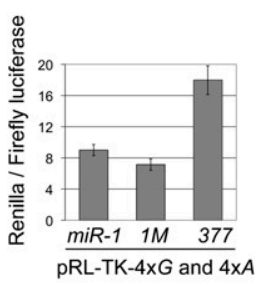

E
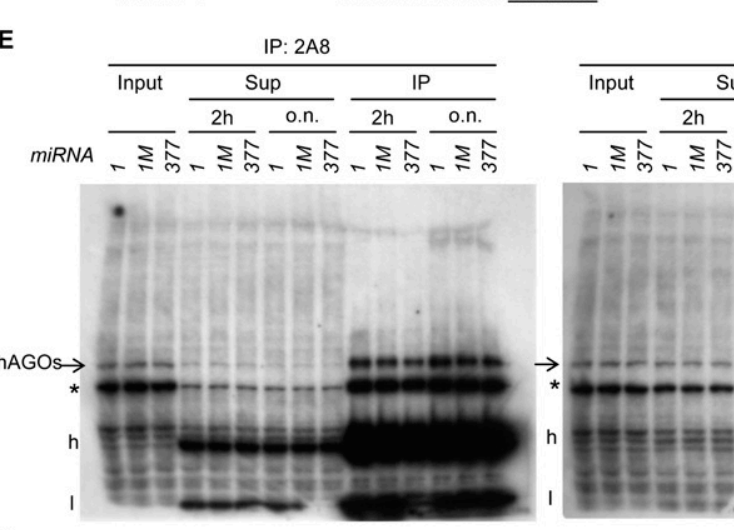

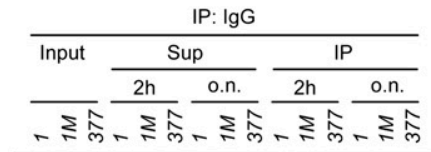

$\mathbf{F}$

Input
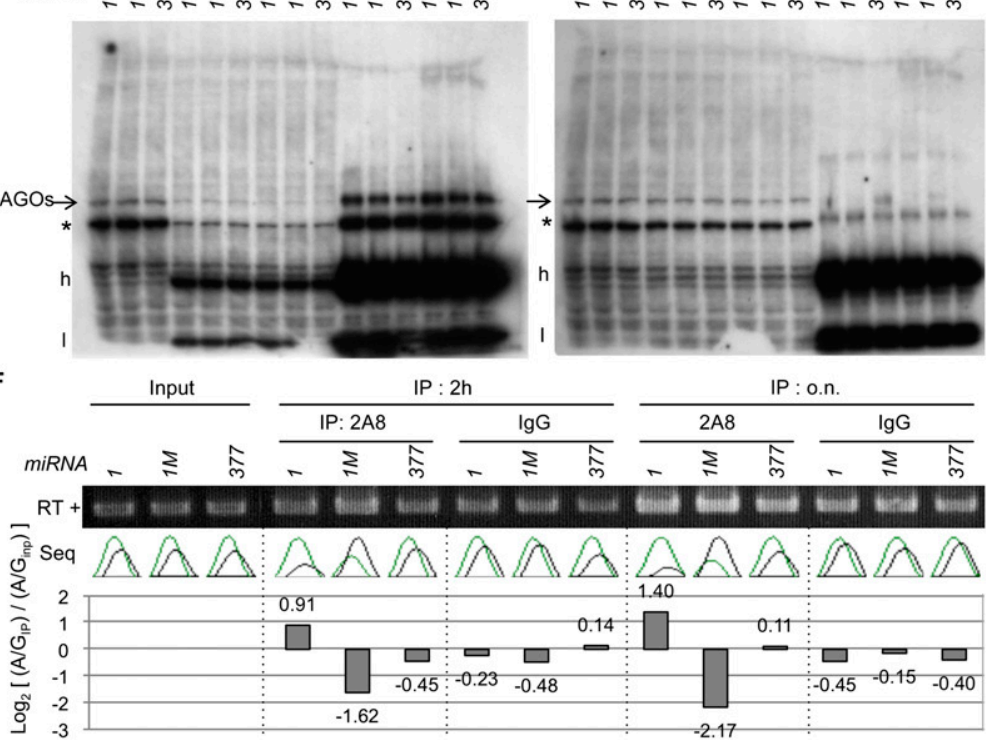

G

pRL-TK-3'A (Texel $A$ allele) HSV-TK $\sqrt{\text { Ren-luc MSTN 3. UTR }}$

pRL-TK-3'G (WT G allele) HSV-TK Ren-IUC MSTN 3' UTR pA

FIGURE 2. (Legend on next page) 


\section{Negligible post-lysis reassociation of RISC complexes with and without cross-linking}

Previous reports indicate that RNA-protein complexes, including miRNP, may form after cell lysis (e.g., Mili and
Steitz 2004; Nelson et al. 2007). Thus, the coimmunoprecipitated targets may reflect the formation of artifacts rather than the occurrence of genuine silencing activity pre-existing in the living cells. As cross-linking might affect the incidence of this phenomenon, we first tested whether our AGO-RIP procedure would also work with fixed samples. As before, we transfected HeLa cells with an equimolar mixture of $p R L-T K-4 x A$ and $p R L-$ $T K-4 x G$ along with either $p c D N A 3-$ $m i R-1$ or $p c D N A 3-m i R-1 M$, yet applied a cross-linking step with formaldehyde $(0.5 \%$, room temperature, $10 \mathrm{~min})$ before cell lysis, and subsequently performed RIP as described above. While we achieved comparable levels of reciprocal allelic imbalance as in the previous experiment, irrespective of fixation or not, we systematically (1) detected lower amounts of AGO protein by Western blotting and (2) obtained less RT-PCR product from the fixed than from the unfixed samples (Supplemental Fig. 2). This suggests that fixation compromises the efficacy of RIP by reducing the affinity of the 2A8 antibody for AGOs and/ or the recovery of intact target RNA as a result of damage endured during fixation or cross-linking reversal (e.g., Masuda et al 1999).

We then evaluated the degree of post-lysis miRNP reassociation and the effect of fixation on it by comparing the level of $A / G$ allelic imbalance in the RIP product obtained from cells that were cotransfected with the $p R L$ $T K-4 x A / 4 x G$ mix and the pre-miRNAexpressing pcDNA3 vectors, with that obtained from a mixture of two cell populations transfected separately with the $p R L-T K-4 x A / 4 x G$ mix or primiRNA-expressing pcDNA3 vectors (Fig. 3A). The cell populations were either fixed or not, mixed, lysed by sonication, and cell extracts subjected to RIP using the same experimental conditions as above. The expected reciprocal allelic imbalance was recapitulated when target- and miRNA-expressing vectors were cotransfected in the same cells. However, when mixing lysates of cell populations transfected separately with either the target- or miRNA-expressing vectors, we observed no evidence of significant allelic imbalance whether with 
A

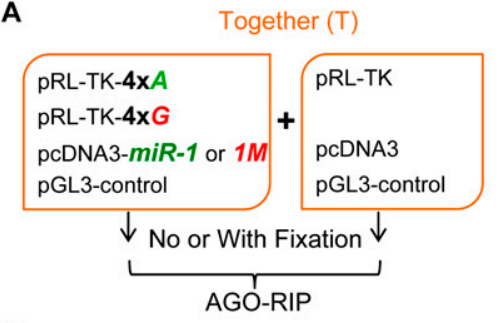

B
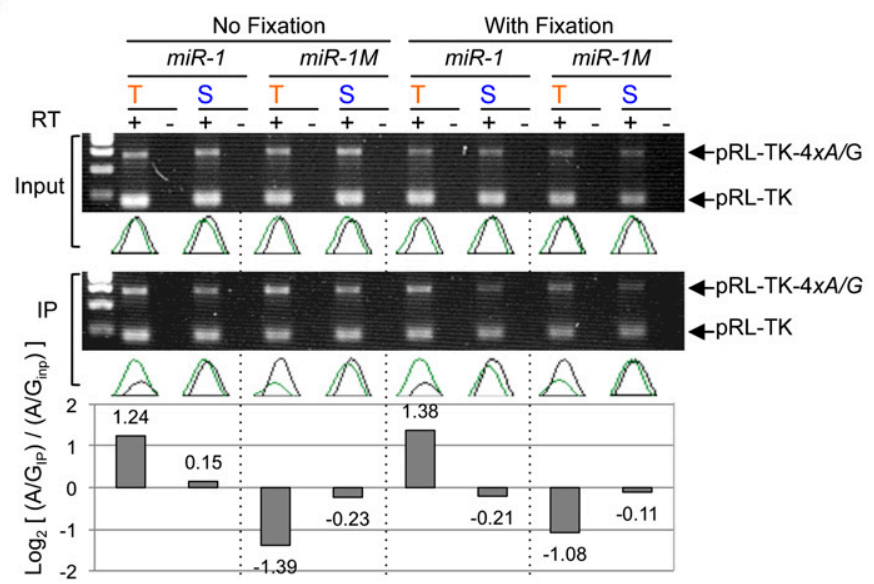

FIGURE 3. (A) Schematic representation of the experiment conducted to evaluate the rate of post-lysis reassociation of the RISC, which might generate artifactual evidence of polymorphic miRNA-mediated gene regulation. Plasmids expressing targets (mix of $p R L-T K-4 x A$ and $p R L-$ $T K-4 x G$ ) and miRNAs (either $p c D N A 3-m i R-1$ or $p c D N A 3-m i R-1 M$ ) were either transfected together (T) or separately $(\mathrm{S})$ in HeLa cells. The plasmid mixes were complemented with matching empty vectors (pRL-TK, pcDNA3) to ensure transfection of equal amounts of DNA, as indicated. Cells were either fixed with formaldehyde $(0.5 \%$, room temperature, $10 \mathrm{~min})$ or not, and complementary cell populations mixed as indicated before proceeding with the AGORIP procedure. $(B)$ Allelic imbalance test demonstrating that post-lysis reassociation of the RISC is negligible in the experimental conditions used. RNA was isolated from the input and IP samples, reverse transcribed (RT+) or not (RT-) using random hexamers, and PCR amplified using primers spanning the tandem insertions. SYBRsafe-stained PCR products after agarose gel eletrophoresis show the RT-PCR products corresponding to the $p R L-T K-4 x A / p R L$ $T K-4 x G$ as well as empty $p R L-T K$ vectors, respectively. The $p R L-T K-4 x A / p R L-T K-4 x G$-derived RT-PCR products were directly sequenced. The electropherograms at the polymorphic site are shown for both input and IP. Peak heights of each allele $(A, G)$ were obtained using Peakpicker. The degree of allelic imbalance was estimated as $\log _{2}$ of the ratio of ratios of $A$ over $G$ peak height, after (IP) and before immunoprecipitation (Input). The $m i R-1 /-1 M$ and $2 A 8$-dependent reciprocal allelic imbalance is observed only when target- and miRNA-expressing vectors are transfected together, not when transfected separately, irrespective of fixation conditions. the wild-type $\left(p R L-T K-3^{\prime} G\right)$ or mutant $\left(p R L-T K-3^{\prime} A\right)$ full-length $(1.5 \mathrm{~Kb})$ MTSN 3'UTR sequence of sheep. When coexpressing $m i R-1$, the Texel mutation was previously shown to cause an $\sim 1.3$ fold reduction of luciferase activity from these vectors (Clop et al. 2006).

HeLa cells were cotransfected with (1) an equimolar mixture of $p R L-T K-$ $3^{\prime} A$ and $p R L-T K-3^{\prime} G$ and (2) either pcDNA3-miR-1, pcDNA3-miR-1M, or pcDNA3-miR-377 (Fig. 2G). RIP analysis was conducted as described above, and RT-PCR products were directly sequenced and analyzed with Peakpicker. The procedure was shown to be quantitative with these amplicons as well (Supplemental Fig. 1B). Using the ratio of immunoprecipitated transcripts with the $A$ versus $G$ allele from cells cotransfected with $p c D N A 3-m i R-377$ as a reference, we observed (1) an approximately twofold enrichment of the mutant $A$ allele when coexpressing $m i R-1$, and (2) an $\sim 1.8$-fold enrichment of the wildtype $G$ allele when coexpressing $m i R-1 M$ (Fig. 2H). A control experiment conducted in parallel with equimolar mixtures of $p R L-T K-4 x A$ and $p R L-T K-4 x G$ yielded $\sim 1$.7-fold and $\sim 2.5$-fold enrichments, respectively (Fig. $2 \mathrm{H}$ ). Thus, although one target site in the 3'UTR of the reporter vector causes a reduction in luciferase activity that is approximately twofold weaker than four tandem copies, the degree of preferential association of the targeted allele with the RISC complex might be less dependent on the number of target sites. or without formaldehyde treatment (Fig. 3B). These results indicate that post-lysis reassociation of RISC complexes does not occur at a significant rate under our experimental conditions.

\section{Luciferase reporter transcripts endowed with the full-length MSTN 3'UTR sequence carrying the Texel mutation are preferentially coimmunoprecipitated by anti-AGO2 antibodies in a miR-1-dependent fashion}

To more closely mimic the actual situation in skeletal muscle of Texel sheep, we repeated our experiments in cell culture using luciferase reporter vectors endowed with either

\section{Endogenous MSTN transcripts carrying the Texel mutation are preferentially coimmunoprecipitated by anti-AGO2 antibodies from skeletal muscle lysates of heterozygous sheep}

Having established suitable conditions for RIP and allelic imbalance testing on cultured cells, we aimed at expanding our method to the analysis of tissue samples as needed to study polymorphic miRNA-mediated regulation in vivo. To that end we collected skeletal muscle samples (longissmus dorsi and semimembranosus) (Fig. 1A) of a 30-wk-old sheep heterozygous for the $g+6223 G-A$ mutation. The MSTN gene is activated in the dermomyotome during fetal development, yet remains expressed in skeletal muscle during the entire lifetime of the organism (McPherron et al. 1997). 
Muscle samples were minced, incubated on ice in $1 \%$ formaldehyde (fixation) or PBS (no fixation), deep frozen, crunched to powder under liquid nitrogen, and subject to the same lysis, RIP, RT-PCR, and sequencing procedures as the cultured cells with minor modifications (see Materials and Methods; Supplemental Fig. 1C).

We first verified whether the 2A8 monoclonal antibody, raised against human AGO2, would be able to immunoprecipitate ovine AGOs proteins. Western blotting performed on input, supernatant, and immunoprecipitate indicated that sheep AGO proteins could indeed be successfully immunopreciptated from skeletal muscle, both with and without cross-linking (Fig. 4A). We then RT-PCR amplified a segment of the MSTN transcripts embracing the $g+6223 G-A$ site. Sequence analysis of the RT-PCR amplicons obtained from input material revealed an average $\sim 1.5$ fold excess of the wild-type allele over the mutant allele. This confirmed our previous findings using PCR-RFLP, and is assumed to reflect miR-1-dependent degradation of mutant mRNA molecules (Clop et al. 2006). On the other hand, in the RT-PCR amplicons obtained from immunoprecipitated material, the mutant allele proved to be present with an average $\sim 1.4$-fold excess (Fig. 4B). This was exactly as predicted assuming that the mutant transcripts would indeed undergo miR-1-dependent inhibition, while the wild-type transcripts would not. To allow for a more rigorous quantitative assessment of our data, we reanalyzed the RT-PCR products using a calibrated hot-stop PCR-RFLP test that takes advantage of an $H p y C H 4 I V$ restriction site destroyed by the Texel mutation (Supplemental Fig. 1D). Levels of mutant transcripts were estimated to be reduced $\sim 1.54$-fold (range: 1.09-1.89) (when compared with the wild-type allele) in input material, yet increased $\sim 1.52$-fold (range: $1.46-1.60$ ) after immunoprecipitation (IP); hence, corresponding to a net enrichment of $\sim 2.35$ by the RIP procedure (Fig. 4C).

To verify the reproducibility of our results, we repeated the experiment on
A
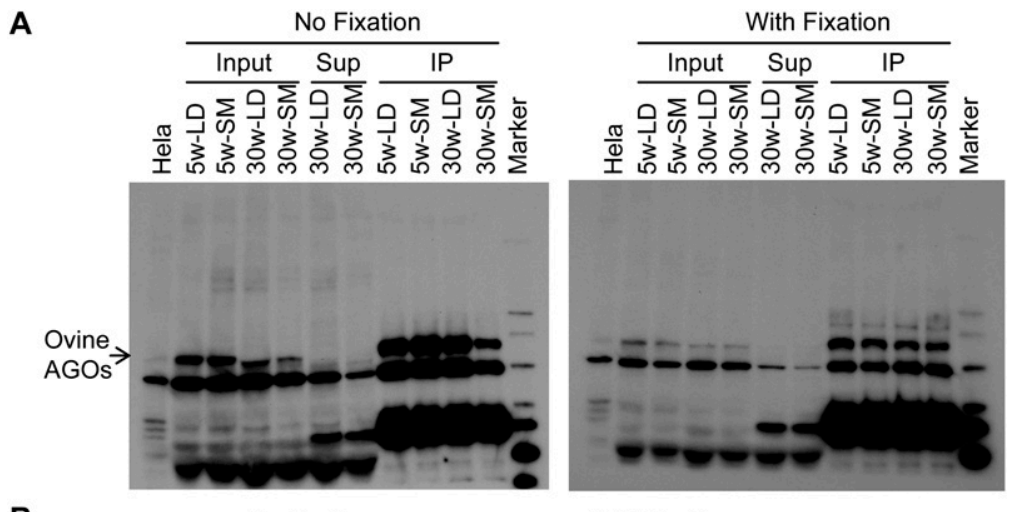

B
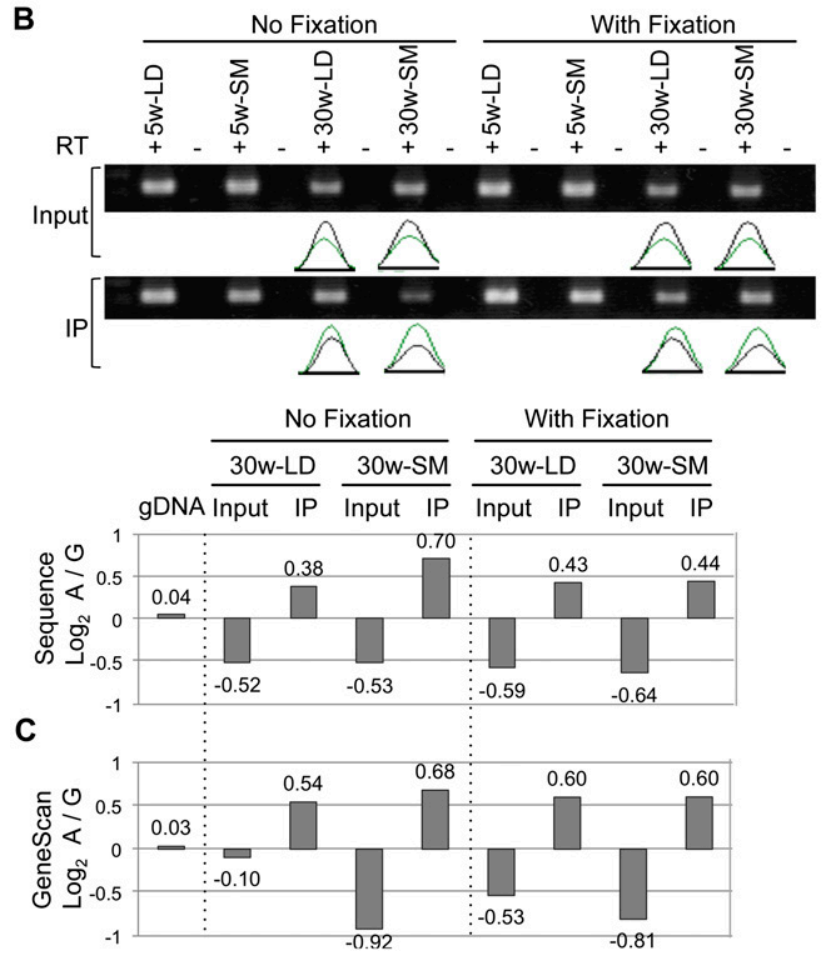

FIGURE 4. (A) Immunoprecipitation of ovine AGO proteins using the $2 \mathrm{~A} 8$ anti-hAGO2 antibody. Skeletal muscle samples (longissimus dorsi and semimembranosus) from a 5- and a 30wk-old sheep were minced, fixed in $1 \%$ formaldehyde or not, deep-frozen, and lysed. Cell lysates were incubated overnight with the $2 \mathrm{~A} 8$ antibody. (Input), supernatant (Sup) after immunoprecipitation, and immunoprecipitates (IP) were loaded on PAGE gels and subjected to Western blotting using the same 2A8 antibody. Enrichment of ovine AGOs in IP samples and depletions in supernatants were observed. (B) Allelic imbalance test performed on the fixed and nonfixed skeletal muscle lysates of the 30 -wk-old animal, heterozygous for the $g+6223 G-A$ mutation (the 5 -wk-old animal was homozygous wild type). RNA was isolated from the input and IP samples, reverse transcribed $(\mathrm{RT}+)$ or not $(\mathrm{RT}-)$ using random hexamers, and PCR-amplified using primers spanning the $g+6223 G-A$ site. SYBRsafe-stained PCR products after agarose gel electrophoresis are shown, and were directly sequenced. The electropherograms at the polymorphic site are shown for both input and IP. Peak heights of each allele $(A, G)$ were obtained using Peakpicker. The degree of allelic imbalance was estimated as $\log _{2}$ of the ratio of $A$ over $G$ peak height. Note the depletion of the mutant $A$ allele in the input as a result of its $m i R-1 /-206-$ dependent degradation, yet its clear enrichment after AGO-RIP, demonstrating its preferential association with the RISC in vivo. $(C)$ Allelic imbalance test using fluorescent PCR-RFLP as alternative. Genomic and RT-PCR products obtained from a heterozygous sheep were labeled by adding a fluorescent primer at the last PCR cycle ("hot stop PCR") (Uejima et al. 2000) and digested with a restriction enzyme cleaving the wild-type but not mutant allele. After capillary gel electrophoresis on an ABI3730 instrument, the mutant and wild-type allele were recognized as fluorescent fragments of, respectively, 253 and $98 \mathrm{bp}$. The areas under the allele-specific peaks were estimated using the Peak Scanner software (Applied Biosystems). The degree of allelic imbalance was estimated as $\log _{2}$ of the ratio of $A$ over $G$ peak area in the RT-PCR product. 
longissmus dorsi and semimembranosus of two $\sim 1$-mo-old heterozygous animals. Supplemental Figure 3 shows the ratio ( $\log _{2}$ scale) of the mutant over wild-type allele in input and immunoprecipitated cDNA (precorrected for the allelic ratio measured from genomic DNA of the same heterozygous animals). Averaged across all samples, the mutant allele was depleted $\sim 2$. 3 -fold in input material, while having $\sim 1.3$-fold excess after IP, corresponding therefore to an approximately threefold net enrichment; thus confirming our previous findings. The level of net enrichment did not differ significantly between muscles and animals (data not shown).

\section{DISCUSSION}

We herein describe a biochemical method aimed at formally testing the effect of DNA sequence polymorphisms in target genes on their association with the RISC complex in vivo. We apply it successfully to the ovine MSTN $g+6223 G-A$ mutation and prove that it creates an illegitimate target site for coexpressed microRNAs (presumably $m i R-1$ and $m i R-206)$, as previously hypothesized based on strong genetic evidence and results of reporter assays (Clop et al. 2006). This is the first example of the formal demonstration of such mutational mechanism in a vertebrate.

The proposed method requires the coimmunoprecipitation (co-IP) of miRNA target genes with components of the RISC complex, corresponding to AGO proteins in this study. IP of miRNA targets has previously been described, including in mammals. However, in most previous reports IP relied on the use of epitope-tagged AGO proteins introduced either in cultured cells by transfection (Easow et al. 2007; Karginov et al. 2007; Hendrickson et al. 2008; Landthaler et al. 2008; Fasanaro et al. 2009; Hong et al. 2009; Verrier et al. 2009) or in transgenic Drosophila (Easow et al., 2007) and C. elegans (Zhang et al. 2007, 2009). In mammals, only six publications describe the co-IP of miRNA target genes with endogenous RISC components (Nelson et al. 2004; Beitzinger et al. 2007; Vasudevan and Steitz 2007; Chi et al. 2009; Tan et al. 2009; Wang et al. 2010). Only one of these, using the high-throughput sequencing of RNAs isolated by cross-linking IP (HITS-CLIP) method (Chi et al. 2009), reported successful miRNA target co-IP from tissue samples, as also achieved in this work. The HITSCLIP method is certainly more specific in coprecipitating miRNA targets than the method described in this work. However, HITS-CLIP is still considerably more laborious and challenging technically. The lower specificity of our approach is effectively compensated for by the combination of RIP with an allelic imbalance test. Most of the experimental noise that one would face when comparing the levels of co-IP of a given target gene between individuals of different genotypes for the polymorphism of interest is effectively neutralized by comparing the behavior of the two alleles in tissue samples from heterozygous individuals. The number of samples needed to convincingly demonstrate even relatively subtle effects (such as the $\sim 2.35$-fold net enrichment in this work) is therefore drastically reduced.

Our method is readily applicable to test the validity of the hypothesized perturbed miRNA-mediated gene regulation put forward for at least 10 human SNPs associated with pathological conditions (for review, see Sethupathy and Collins 2008).

In combination with high-throughput sequencing approaches (e.g., HIST-CLIP) (Chi et al. 2009), it could be extended to systematic screening for common and rare SNPs that might operate in a similar manner. As an example, it could advantageously be combined with the experimental design recently put forward by Kim and Bartel (2009) in mice. While the allelic imbalance sequencing (AISEQ) procedure applied in this study unambiguously demonstrated that a significant proportion of SNPs altering predicted miRNA target sites affected miRNA-mediated regulation of the target genes, it was not effective at pinpointing the actual culprit polymorphisms. This is due to the fact that allelically imbalanced transcript levels can result from several phenomena other than perturbed miRNA-mediated gene regulation. Targeting transcripts associated with components of the silencing machinery renders the approach described in this work more discriminating.

Protocol optimization using reporter assays yielded some unexpected findings worth mentioning. The first finding is that while $m i R-1$ and $m i R-1 M$ expression caused approximately threefold down-regulation of the luciferase activity from the $p R L-T K-4 x A$ and $p R L-T K-4 x G$ constructs, respectively, both miRNAs used under similar conditions caused a more than twofold down-regulation of an equimolar mix of $p R L-T K-4 x A+p R L-T K-4 x G$, whereas less than twofold was expected (Fig. 2C,D). The repressions observed for the equimolar mix were significantly lower than expected given the observed down-regulation of the isolated constructs $(P=0.03$ for $m i R 1$, and $P=0.02$ for miR1M) (see Supplemental Methods). If confirmed, this would suggest that despite the corresponding seed mismatches, $m i R-1$ and $m i R-1 M$ are able to target $p R L-T K-4 G$ and $p R L-T K-4 x A$, respectively, albeit weakly. The second finding is that while going from four $(p R L-T K-4 x A)$ to one $\left(p R L-T K-3^{\prime} A\right) m i R-1$ target site in the $3^{\prime} \mathrm{UTR}$ of reporter vectors reduced the effect of the $g+6223 G-A$ SNP on luciferase activity approximately twofold, its effect on the degree of RIP-induced allelic imbalance seemed less. While we appreciate that a more rigorous analysis is needed to support this assertion (including the characterization of the dynamic range of our approach), if confirmed, our observation would suggest that the smaller effect of one versus four target sites on gene expression is not as much due to a lower occupancy of the target sites by the RISC complex, but rather by the fact that several RISC complexes are needed per target gene to effectively block translation. 
Thus, the previously described cooperative effect of multiple target sites would operate not at the level of RISC binding to the target gene, but rather at the level of its interference with the translational machinery.

\section{MATERIALS AND METHODS}

\section{Cell culture, transfection, and fixation}

HeLa celles $\left(2 \times 10^{6}\right.$ cells $)$ were plated in a $10-\mathrm{cm}$ dish $24 \mathrm{~h}$ prior to transfection and then transfected with $4.8 \mu \mathrm{g}$ each of two reporter vectors endowed with either target or nontarget sequence in the 3'UTR of pRL-TK vector (Promega) along with $12 \mu \mathrm{g}$ of one of the miRNA expression vectors in pcDNA3.1 (Invitrogen) and $0.3 \mu \mathrm{g}$ of pGL3-control vector (Promega) using $45 \mathrm{uL}$ of Lipofectamine 2000 (Invitrogen) according to the manufacturer's instructions. All plasmid constructs except for the $p c D N A-m i R-$ $1 M$ were described previously (Clop et al. 2006). The $p c D N A-m i R-$ $1 M$ was made from $p c D N A 3-m i R-1$ by mutagenizing the $T$ at position 6 of $m i R-1$ into $C$ using the QuikChange II Site-Directed Mutagenesis kit (Stratagene). At 24 h post-transfection, cells were washed twice with ice-cold PBS supplied with complete protease inhibitors (Roche) and harvested into tubes using a cell scraper. When indicated, cells were fixed before harvesting on a plate with $0.5 \%$ formaldehyde at room temperature for $10 \mathrm{~min}$, followed by incubating with $0.125 \mathrm{M}$ glycine at room temperature for $5 \mathrm{~min}$.

\section{AGO-RIP and luciferase assay}

Immunoprecipitation was carried out essentially as described by Mourelatos et al. (2002). All manipulations were performed at $4^{\circ} \mathrm{C}$ or on ice. Briefly, HeLa cells from a $10-\mathrm{cm}$ dish were suspended in $850 \mu \mathrm{L}$ of ice-cold lysis buffer $(20 \mathrm{mM}$ Tris- $\mathrm{HCl}$ at $\mathrm{pH} 7.4,200 \mathrm{mM}$ $\mathrm{NaCl}, 2.5 \mathrm{mM} \mathrm{MgCl}, 0.25 \%$ Igepal CA-630, $1.5 \mu \mathrm{L} / \mathrm{mL}$ RNasin [Promega], $1.5 \mu \mathrm{L} / \mathrm{mL}$ SUPERaseIn [Ambion], 1X EDTA-free complete protease inhibitors [Roche]), and lysed with brief sonications using Bioruptor (Diagenode) for $30 \mathrm{sec}$, three times, with a high power setting (or low when indicated). Nuclei and debris were pelleted at $10,000 \mathrm{~g}$ for $10 \mathrm{~min}$. Part of the supernatant $(\sim 5$ $\mu \mathrm{L})$ was used to measure luciferase activities using the DualLuciferase Reporter Assay system (Promega). The rest was precleared once using $12.5 \mu \mathrm{L}$ of Protein G beads (Invitrogen or Santa Cruz Biotechnology) coated with nonimmune mouse IgG and tRNA (Invirogen) and once with noncoated Protein G beads for $1 \mathrm{~h}$ with a gentle rotation, followed by centrigation at $10,000 \mathrm{~g}$ for 10 min. Part of the supernatant $(\sim 40 \mu \mathrm{L})$ was kept on ice as an "input" sample. The precleared lysate was immunoprecipitated with $25 \mu \mathrm{L}$ of Protein G beads precoated with either $20 \mu \mathrm{L}$ of anti hAGO2 monoclonal antibody 2A8, generously supplied by $\mathrm{Z}$. Mourelatos (University of Pennsylvania School of Medicine; now available from Diagenode), or $10 \mu \mathrm{g}$ of mouse IgG (Invitrogen) as a negative control, with gentle rotation overnight. The beads were pelleted at $1000 \mathrm{~g}$ for $1 \mathrm{~min}$. The supernatant $(\sim 40 \mu \mathrm{L})$ was kept on ice as a "supernatant" sample. The beads were then washed twice with $1 \mathrm{~mL}$ of the lysis buffer for $10 \mathrm{~min}$ with gentle rotation and once by putting the beads on top of $11 \mathrm{~mL}$ of the lysis buffer. The beads were collected by centrifugation at $500 \mathrm{~g}$ for $2 \mathrm{~min}$. One-tenth of the beads were kept as an "IP" sample for Western blotting. RNA was isolated from the rest using either TRIzol LS (Invitrogen) followed by ethanol precipitation with sodium acetate and GlycoBlue (Ambion) or RNeasy FFPE RNA isolation kit (Qiagen) and treated with a Turbo DNA free kit (Ambion) to remove DNA contamination.

\section{AGO-RIP from skeletal muscle}

Skeletal muscle samples (longissmus dorsi and semimembranosus) of a 30-wk-old sheep heterozygous for the $g+6223 G-A$ mutation and a 5 -wk-old sheep homozygous for the wild-type allele were collected. The tissue was cut into small pieces and kept for $\sim 2 \mathrm{~h}$ either in icecold PBS with proteinase inhibitors (nonfixed samples) or in icecold $1 \%$ buffer-saturated formalin (SIGMA) (fixed samples). One gram of sample was grinded with a pestle and mortar in the presence of liquid nitrogen (this step can be omitted for nonfixed samples) and homogenized with a potter-elvehjem glass-Teflon homogenizer in $4 \mathrm{~mL}$ of ice-cold lysis buffer. Large cell debris were eliminated by filtering the homogenate through $100-\mu \mathrm{m}$ cell strainers. AGO-RIP was performed as above.

\section{Measuring $g+6723 G-A$ allelic imbalance at the RNA level using sequencing and the GeneScan system}

cDNA was synthesized using RevertAid H Minus Reverse transcriptase (Fermentas) with random hexamer either with (RT+) or without reverse-transcriptase (RT-). PCR was performed using Phusion High-Fidlity DNA polymerase (Finnzymes) with primers pRL-f3: TGTTGAAGGTGCCAAGAAGTTTCC and pG3L-r1: GTA TCTTATCATGTCTGCTCGAAG (annealing temperature, $64^{\circ} \mathrm{C}$ ) to amplify inserts (521 bp) from $p R L-T K-4 x A$ - and G-derived transcripts. For amplifying the polymorphic site on the ovine MSTN 3'UTR (253 bp), primers Mstn-3UTR-UP1: GGTCTTAAAACTC CATATGCAAA and Mstn-3UTR-DN1: CAATTTGTAAGATAC CATCAGTT (annealing $55^{\circ} \mathrm{C}$ ) were used. cDNA (RT+) specific amplification was confirmed by agarose gel electrophoresis and SYBR Safe DNA gel staining (Invitrogen) of the PCR product. The rest of the PCR product was purified through a MultiScreen PCRu96 filter plate (Millipore) and sequenced using BigDye Terminator v3.1 Cycle Sequencing kits and a 3730 DNA Analyzer (Applied Biosystems) with either the pRL-f3 or Mstn-3UTR-DN1 primers. Peak heights at the polymorphic site were quantified using the PeakPicker software (Ge et al. 2005). Allelic imbalance was estimated as the ratio of peak height of the $A$ allele (Texel mutant) over the $G$ allele (wild type). When indicated, it was normalized with the input sample ratio. To quantify the allelic imbalance more rigorously, a hot-stop PCR-RFLP method was performed as previously described by Clop et al. (2006) with some modifications. Briefly, the PCR product from the ovine MSTN 3'UTR (253 bp) was labeled by adding a $5^{\prime}$ fluorescent FAM-Mstn-3UTR-DN1 primer at the final cycle of the PCR, purified using a QIAquick PCR Purification kit (Qiagen), digested with a restriction enzyme HpyCH4IV cleaving the wild-type $G$ allele into a 98-bp fragment, but not the Texel $A$ allele, and intensities of the 253-bp (Texel allele) and 98-bp (wildtype allele) fragments were detected using the GeneScan system and the 3730 DNA Analyzer (Applied Biosystems). Peak height and area were quantified using the Peak Scanner software (Applied Biosystems). Calibration curves were made using data obtained by mixing varying amounts of either plasmid DNAs ( $p R L-T K-4 x A$ and $-4 x G, p R L-T K-3^{\prime} A$ and $-3^{\prime} G$ ) or genomic DNAs from $A A$ and $G G$ animals. 


\section{Western blotting}

Samples were mixed with an equal volume of $2 \mathrm{X}$ Laemmli SDS buffer, heated for $3 \mathrm{~min}$ at $100^{\circ} \mathrm{C}$, separated on a $3 \%-8 \%$ Tris acetate NuPAGE gel with 1X TA SDS running buffer (Invitrogen). Proteins were transferred onto a PDVF membrane (Invitrogen), incubated with the $2 \mathrm{~A} 8$ monoclonal antibody (1:300), and detected with horseradish peroxidase-coupled anti-mouse IgG antidbody (1:50000, Amersham) using the ECL Advance Western Blotting Detection kit (Amersham).

\section{SUPPLEMENTAL MATERIAL}

Supplemental material can be found at http://www.rnajournal.org.

\section{ACKNOWLEDGMENTS}

We are very grateful to Dr. Zissimos Mourelatos for generously providing us with the $2 \mathrm{~A} 8$ monoclonal antibody and for helpful comments on our results. We thank Arnaud Sartelet, Dimitri Pirottin, and Romi Pena for the support of the sampling procedure and the GIGA facility for sequencing. This work was funded by grants from the Belgian Fond National de la Recherche Scientifique, the Politique Scientifique Fédérale (PAI-Gene Func), the Communauté Française de Belgique (ARC-Mirage), and the University of Liège. C.C. is Chercheur Qualifié from the Belgian Fond National de la Recherche Scientifique.

Received February 16, 2010; accepted June 10, 2010.

\section{REFERENCES}

Abelson J, Kwan K, O’Roak B, Baek D, Stillman A, Morgan T, Mathews C, Pauls D, Rasin M, Gunel M, et al. 2005. Sequence variants in SLITRK1 are associated with Tourette's syndrome. Science 310: 317-320.

Adams B, Furneaux H, White B. 2007. The micro-ribonucleic acid (miRNA) miR-206 targets the human estrogen receptor- $\alpha$ (ER $\alpha)$ and represses $\mathrm{ER} \alpha$ messenger RNA and protein expression in breast cancer cell lines. Mol Endocrinol 21: 1132-1147.

Beetz C, Schüle R, Deconinck T, Tran-Viet K, Zhu H, Kremer B, Frints S, van Zelst-Stams W, Byrne P, Otto S, et al. 2008. REEP1 mutation spectrum and genotype/phenotype correlation in hereditary spastic paraplegia type 31. Brain 131: 1078-1086.

Beitzinger M, Peters L, Zhu J, Kremmer E, Meister G. 2007. Identification of human microRNA targets from isolated argonaute protein complexes. RNA Biol 4: 76-84.

Brendle A, Lei H, Brandt A, Johansson R, Enquist K, Henriksson R, Hemminki K, Lenner P, Forsti A. 2008. Polymorphisms in predicted microRNA-binding sites in integrin genes and breast cancer: ITGB4 as prognostic marker. Carcinogenesis 29: 1394-1399.

Chen K, Rajewsky N. 2007. The evolution of gene regulation by transcription factors and microRNAs. Nat Rev Genet 8: 93-103.

Chi S, Zang J, Mele A, Darnell R. 2009. Argonaute HITS-CLIP decodes microRNA-mRNA interaction maps. Nature 460: 479486.

Chin LJ, Ratner E, Leng S, Zhai R, Nallur S, Babar I, Muller RU, Straka E, Su L, Burki EA, et al. 2008. A SNP in a let-7 microRNA complementary site in the KRAS $3^{\prime}$ untranslated region increases non-small cell lung cancer risk. Cancer Res 68: 8535-8540.

Clop A, Marcq F, Takeda H, Pirottin D, Tordoir X, Bibé B, Bouix J, Caiment F, Elsen J, Eychenne F, et al. 2006. A mutation creating a potential illegitimate microRNA target site in the myostatin gene affects muscularity in sheep. Nat Genet 38: 813-818.
Easow G, Teleman A, Cohen S. 2007. Isolation of microRNA targets by miRNP immunopurification. RNA 13: 1198-1204.

Fasanaro P, Greco S, Lorenzi M, Pescatori M, Brioschi M, Kulshreshtha R, Banfi C, Stubbs A, Calin G, Ivan M, et al. 2009. An integrated approach for experimental target identification of hypoxia-induced miR-210. J Biol Chem 284: 35134-35143.

Friedman R, Farh K, Burge C, Bartel D. 2009. Most mammalian mRNAs are conserved targets of microRNAs. Genome Res 19: 92-105.

Ge B, Gurd S, Gaudin T, Dore C, Lepage P, Harmsen E, Hudson T, Pastinen T. 2005. Survey of allelic expression using EST mining. Genome Res 15: 1584-1591.

Hendrickson D, Hogan D, Herschlag D, Ferrell J, Brown P. 2008. Systematic identification of mRNAs recruited to argonaute 2 by specific microRNAs and corresponding changes in transcript abundance. PLoS ONE 3: e2126. doi: 10.1371/journal.pone.0002126.

Hiard S, Charlier C, Coppieters W, Georges M, Baurain D. 2010. Patrocles: A database of polymorphic miRNA-mediated gene regulation in vertebrates. Nucleic Acids Res 38: D640-D651.

Hong X, Hammell M, Ambros V, Cohen S. 2009. Immunopurification of Agol miRNPs selects for a distinct class of microRNA targets. Proc Natl Acad Sci 106: 15085-15090.

Jensen K, Covault J, Conner T, Tennen H, Kranzler H, Furneaux H. 2009. A common polymorphism in serotonin receptor $1 \mathrm{~B}$ mRNA moderates regulation by miR-96 and associates with aggressive human behaviors. Mol Psychiatry 14: 381-389.

Kapeller J, Houghton L, Mönnikes H, Walstab J, Möller D, Bönisch H, Burwinkel B, Autschbach F, Funke B, Lasitschka F, et al. 2008. First evidence for an association of a functional variant in the microRNA-510 target site of the serotonin receptor-type 3E gene with diarrhea predominant irritable bowel syndrome. Hum Mol Genet 17: 2967-2977.

Karginov F, Conaco C, Xuan Z, Schmidt B, Parker J, Mandel G, Hannon G. 2007. A biochemical approach to identifying microRNA targets. Proc Natl Acad Sci 104: 19291-19296.

Kim J, Bartel D. 2009. Allelic imbalance sequencing reveals that singlenucleotide polymorphisms frequently alter microRNA-directed repression. Nat Biotechnol 27: 472-477.

Landi D, Gemignani F, Naccarati A, Pardini B, Vodicka P, Vodickova L, Novotny J, Försti A, Hemminki K, Canzian F, et al. 2008. Polymorphisms within micro-RNA-binding sites and risk of sporadic colorectal cancer. Carcinogenesis 29: 579-584.

Landthaler M, Gaidatzis D, Rothballer A, Chen P, Soll S, Dinic L, Ojo T, Hafner M, Zavolan M, Tuschl T. 2008. Molecular characterization of human Argonaute-containing ribonucleoprotein complexes and their bound target mRNAs. RNA 14: 2580-2596.

Lewis M, Quint E, Glazier A, Fuchs H, De Angelis M, Langford C, van Dongen S, Abreu-Goodger C, Piipari M, Redshaw N, et al. 2009. An ENU-induced mutation of miR-96 associated with progressive hearing loss in mice. Nat Genet 41: 614-618.

Lv K, Guo Y, Zhang Y, Wang K, Jia Y, Sun S. 2008. Allele-specific targeting of hsa-miR-657 to human IGF2R creates a potential mechanism underlying the association of ACAA-insertion/deletion polymorphism with type 2 diabetes. Biochem Biophys Res Commun 374: 101-105.

Masuda N, Ohnishi T, Kawamoto S, Monden M, Okubo K. 1999. Analysis of chemical modification of RNA from formalin-fixed samples and optimization of molecular biology applications for such samples. Nucleic Acids Res 27: 4436-4443.

McPherron A, Lawler A, Lee S. 1997. Regulation of skeletal muscle mass in mice by a new TGF- $\beta$ superfamily member. Nature 387: 83-90.

Mencía A, Modamio-Høybjør S, Redshaw N, Morín M, Mayo-Merino F, Olavarrieta L, Aguirre L, del Castillo I, Steel K, Dalmay T, et al. 2009. Mutations in the seed region of human miR-96 are responsible for nonsyndromic progressive hearing loss. Nat Genet 41: 609-613.

Mili S, Steitz J. 2004. Evidence for reassociation of RNA-binding proteins after cell lysis: Implications for the interpretation of immunoprecipitation analyses. RNA 10: 1692-1694. 
Mishra P, Humeniuk R, Longo-Sorbello G, Banerjee D, Bertino J. 2007. A miR-24 microRNA binding-site polymorphism in dihydrofolate reductase gene leads to methotrexate resistance. Proc Natl Acad Sci 104: 13513-13518.

Mourelatos Z, Dostie J, Paushkin S, Sharma A, Charroux B, Abel L, Rappsilber J, Mann M, Dreyfuss G. 2002. miRNPs: A novel class of ribonucleoproteins containing numerous microRNAs. Genes Dev 16: $720-728$.

Nelson P, Hatzigeorgiou A, Mourelatos Z. 2004. miRNP:mRNA association in polyribosomes in a human neuronal cell line. RNA 10: 387-394.

Nelson P, De Planell-Saguer M, Lamprinaki S, Kiriakidou M, Zhang P, O’Doherty U, Mourelatos Z. 2007. A novel monoclonal antibody against human Argonaute proteins reveals unexpected characteristics of miRNAs in human blood cells. RNA 13: 1787-1792.

Sethupathy P, Collins F. 2008. MicroRNA target site polymorphisms and human disease. Trends Genet 24: 489-497.

Sethupathy P, Borel C, Gagnebin M, Grant G, Deutsch S, Elton T, Hatzigeorgiou A, Antonarakis S. 2007. Human microRNA-155 on chromosome 21 differentially interacts with its polymorphic target in the AGTR1 3' untranslated region: A mechanism for functional single-nucleotide polymorphisms related to phenotypes. Am J Hum Genet 81: 405-413.

Tan Z, Randall G, Fan J, Camoretti-Mercado B, Brockman-Schneider R, Pan L, Solway J, Gern J, Lemanske R, Nicolae D, et al 2007. Allele-specific targeting of microRNAs to HLA-G and risk of asthma. Am J Hum Genet 81: 829-834.

Tan L, Seinen E, Duns G, de Jong D, Sibon O, Poppema S, Kroesen B, Kok K, van den Berg A. 2009. A high throughput experimental approach to identify miRNA targets in human cells. Nucleic Acids Res 37: e137. doi: 10.1093/nar/gkp715.

Uejima H, Lee M, Cui H, Feinberg A. 2000. Hot-stop PCR: A simple and general assay for linear quantitation of allele ratios. Nat Genet 25: $375-376$.

Vasudevan S, Steitz J. 2007. AU-rich-element-mediated up-regulation of translation by FXR1 and Argonaute 2. Cell 128: 1105-1118.

Verrier J, Lau P, Hudson L, Murashov A, Renne R, Notterpek L. 2009. Peripheral myelin protein 22 is regulated post-transcriptionally by miRNA-29a. Glia 57: 1265-1279.

Wang G, van der Walt J, Mayhew G, Li Y, Züchner S, Scott W, Martin E, Vance J. 2008. Variation in the miRNA-433 binding site of FGF20 confers risk for Parkinson disease by overexpression of $\alpha$-synuclein. Am J Hum Genet 82: 283-289.

Wang W, Wilfred B, Hu Y, Stromberg A, Nelson P. 2010. AntiArgonaute RIP-Chip shows that miRNA transfections alter global patterns of mRNA recruitment to microribonucleoprotein complexes. RNA 16: 394-404.

Zhang L, Ding L, Cheung TH, Dong M-Q, Chen J, Sewell AK, Liu X, Yates JR III, Han M. 2007. Systematic identification of C. elegans miRISC proteins, miRNAs, and mRNA targets by their interactions with GW182 proteins AIN-1 and AIN-2. Mol Cell 28: 598-613.

Zhang L, Hammell M, Kudlow B, Ambros V, Han M. 2009. Systematic analysis of dynamic miRNA-target interactions during C. elegans development. Development 136: 3043-3055.

Züchner S, Wang G, Tran-Viet K, Nance M, Gaskell P, Vance J, Ashley-Koch A, Pericak-Vance M. 2006. Mutations in the novel mitochondrial protein REEP1 cause hereditary spastic paraplegia type 31. Am J Hum Genet 79: 365-369. 

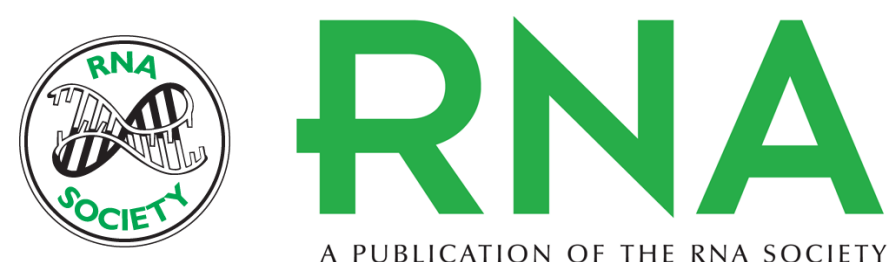

A PUBLICATION OF THE RNA SOCIETY

\section{Demonstrating polymorphic miRNA-mediated gene regulation in vivo: Application to the $g+6223 G \rightarrow A$ mutation of Texel sheep}

Haruko Takeda, Carole Charlier, Frédéric Farnir, et al.

RNA 2010 16: 1854-1863 originally published online August 2, 2010

Access the most recent version at doi:10.1261/rna.2131110

\section{Supplemental http://rnajournal.cshlp.org/content/suppl/2010/07/12/rna.2131110.DC1 \\ Material}

References This article cites 44 articles, 16 of which can be accessed free at: http://rnajournal.cshlp.org/content/16/9/1854.full.html\#ref-list-1

\section{License}

Email Alerting Receive free email alerts when new articles cite this article - sign up in the box at the Service top right corner of the article or click here.

\section{III!"II Providing Precise Solutions tor your research.}

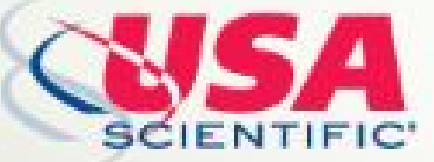

To subscribe to $R N A$ go to:

http://rnajournal.cshlp.org/subscriptions 